\title{
Reconstruction of Spatial Thermal Gradient Encoded in Thermosensory Neuron AFD in Caenorhabditis elegans
}

\author{
Yuki Tsukada, ${ }^{1}$ Masataka Yamao, ${ }^{2}$ Honda Naoki, ${ }^{2}$ Tomoyasu Shimowada, ${ }^{1}$ Noriyuki Ohnishi, ${ }^{1}$ Atsushi Kuhara, ${ }^{3}$ \\ Shin Ishii, ${ }^{2}$ and Ikue Mori ${ }^{1}$ \\ ${ }^{1}$ Group of Molecular Neurobiology, Division of Biological Science, Graduate School of Science, Nagoya University, Furo-cho, Chikusa-ku, Nagoya 464-8602, \\ Japan, ${ }^{2}$ Department of Systems Science, Graduate School of Informatics, Kyoto University, Yoshida-honmachi, Sakyo-ku, Kyoto 606-8501, Japan, \\ ${ }^{3}$ Laboratory of Molecular and Cellular Regulation, Faculty of Science and Engineering, Institute for Integrative Neurobiology, Konan University, \\ Higashinada-ku, Kobe 658-8501, Japan
}

During navigation, animals process temporal sequences of sensory inputs to evaluate the surrounding environment. Thermotaxis of Caenorhabditis elegans is a favorable sensory behavior to elucidate how navigating animals process sensory signals from the environment. Sensation and storage of temperature information by a bilaterally symmetric pair of thermosensory neurons, AFD, is essential for the animals to migrate toward the memorized temperature on a thermal gradient. However, the encoding mechanisms of the spatial environment with the temporal AFD activity during navigation remain to be elucidated. Here, we show how the AFD neuron encodes sequences of sensory inputs to perceive spatial thermal environment. We used simultaneous calcium imaging and tracking system for a freely moving animal and characterized the response property of AFD to the thermal stimulus during thermotaxis. We show that AFD neurons respond to shallow temperature increases with intermittent calcium pulses and detect temperature differences with a critical time window of $20 \mathrm{~s}$, which is similar to the timescale of behavioral elements of $C$. elegans, such as turning. Convolution of a thermal stimulus and the identified response property successfully reconstructs AFD activity. Conversely, deconvolution of the identified response kernel and AFD activity reconstructs the shallow thermal gradient with migration trajectory, indicating that AFD activity and the migration trajectory are sufficient as the encoded signals for thermal environment. Our study demonstrates bidirectional transformation between environmental thermal information and encoded neural activity.

Key words: C. elegans; imaging; reconstruction; response function; thermosensory neuron; tracking

Significance Statement

Deciphering how information is encoded in the nervous system is an important challenge for understanding the principles of information processing in neural circuits. During navigation behavior, animals transform spatial information to temporal patterns of neural activity. To elucidate how a sensory system achieves this transformation, we focused on a thermosensory neuron in Caenorhabditis elegans called AFD, which plays a major role in a sensory behavior. Using tracking and calcium imaging system for freely moving animals, we identified the response property of the AFD. The identified response property enabled us to reconstruct both neural activity from a temperature stimulus and a spatial thermal environment from neural activity. These results shed light on how a sensory system encodes the environment.

\section{Introduction}

Evaluation of the surrounding environment is a vital ability for living organisms. When animals migrate through their environ-

\footnotetext{
Received July 28, 2015; revised Dec. 12, 2015; accepted Dec. 31, 2015.

Author contributions:Y.T., H.N.,S.I, and I.M. designed research;Y.T. performed research;Y.T., T.S., N.O., and A.K. contributed unpublished reagents/analytic tools; Y.T., M.Y., and H.N. analyzed data; Y.T., S.I., and I.M. wrote the paper.

This work was supported by Japan Society for the Promotion of Science Research Funds Grants 24700302 and 23115507, Core Research for Evolutional Science and Technology/Japan Science and Technology Agency, Strategic Research Program for Brain Sciences by Ministry of Education, Culture, Sports, Science, and Technology (MEXT) and Japan Agency for Medical Research and Development (AMED), and the Platform Project for
}

ment, sensory systems convert external information into neural activities as temporally encoded signals. Depending on the encoded signals, animals organize sequences of movement with proper timing to migrate to preferred environments. Elucidating the neuronal mechanisms by which temporal activities of sensory neurons generated a response to environmental input is crucial for understanding the neural code to express and convey the

Supporting in Drug Discovery and Life Science Research by MEXT and AMED. We thank the Caenorhabditis Genetics Center and Dr. S. Xu for strains and M. Vergassola for the initial discussion about response kernel. The authors declare no competing financial interests.

This article is freely available online through the J Neurosci Author Open Choice option. 
information of external world (Rieke et al., 1999). Sensory signals and motor regulation often interact with each other (GomezMarin et al., 2011). Therefore, assessing the sensory encoding of a spatial field during navigation behavior is best conducted in freely behaving animals, in which any sensorimotor feedback is maintained.

Thermotaxis of Caenorhabditis elegans provides a useful model to elucidate how a natural environment is encoded in a sensory system during navigation behavior, because the environmental stimulus can be measured quantitatively and the circuit controlling the behavior is identified and is compact (Hedgecock and Russell, 1975; Mori and Ohshima, 1995). Laser ablation and genetic perturbation of a pair of sensory neurons, AFD, severely disrupts thermotaxis, suggesting that AFD plays a critical role in thermotaxis (Mori and Ohshima, 1995; Satterlee et al., 2001). Calcium imaging shows that AFD responds to a thermal ramp around the cultivation temperature, and this response range of AFD can be changed on shifting the cultivation temperature, suggesting that AFD stores the acclimation temperature (Kimura et al., 2004; Clark et al., 2006). Electrophysiology shows that AFD neurons respond to both increases and decreases of temperature around their responding range with short-term adaptation (Ramot et al., 2008). At the behavioral level, temperature increases modulate the frequency of turning, which is assumed to control thermotaxis with a strategy called biased random walk (Ryu and Samuel, 2002; Zariwala et al., 2003; Luo et al., 2014); additional behavioral strategies, such as weather-vaning strategy (Iino and Yoshida, 2009), could also contribute to thermotaxis.

In this study, we estimated the response function of AFD from data acquired with our tracking and calcium imaging system. The estimated response functions indicated the differential detection mechanism of the AFD neurons, which includes a fast positive response to temperature change, followed by slow adaptation. By estimating the response functions for several cultivation conditions, we showed that the AFD response property was independent of cultivation conditions except at the operating range that is defined by cultivation temperature as a modifiable responsive range to thermal stimuli. We added a nonlinear character to our AFD response model to incorporate the response dependency on the operating range. The constructed response model enabled us to reconstruct AFD activity from the observed temperature input and, conversely, the thermal environment from the observed AFD activity and the migration trajectory. Given the reconstructed activities and thermal environments are highly correlated to actual neuronal activities and the thermal environment, we verified that our model captured the mechanism by which AFD decodes the thermal environment during navigation. These results provide a systems-level understanding of how the nervous system recognizes and processes environmental information during navigation.

\section{Materials and Methods}

Strains and transgenic animals

Wild-type N2 Bristol was obtained from the Caenorhabditis Genetics Center (CGC), and lite-1 (xu7) was a gift from S. Xu (University of

Correspondence should be addressed to Ikue Mori, Group of Molecular Neurobiology, Division of Biological Science, Graduate School of Science, Nagoya University, Furo-cho, Chikusa-ku, Nagoya 464-8602, Japan. E-mail: m46920a@nucc.cc.nagoya-u.ac.jp.

DOI:10.1523/JNEUROSCI.2837-15.2016

Copyright $@ 2016$ Tsukada et al.

This is an Open Access article distributed under the terms of the Creative Commons Attribution License Creative Commons Attribution 4.0 International, which permits unrestricted use, distribution and reproduction in any medium provided that the original work is properly attributed.
Michigan, Ann Arbor, MI). All worms were hermaphrodites and cultivated on OP50 as bacterial food using standard techniques (Brenner, 1974). Microinjection of DNA was performed according to standard protocols (Mello et al., 1991). The following strains were injected with DNA at the described concentrations: IK847 lite-1(xu7);njEx358

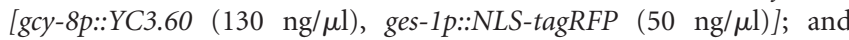
IK2230 lite-1(xu7);njEx662[gcy-5p::YC3.60 (100 ng/ $\mu 1), g c y-5 p:: T a g R F P$ (40 ng/ $\mu \mathrm{l})]$.

\section{Calcium imaging of freely moving animals}

We used the lite-1 (xu7) background to minimize photo avoidance during free migration. The FRET-based calcium probe yellow cameleon 3.60 was driven by the $g c y-8$ or $g c y-5$ promoters to express in AFD, or ASER, respectively. For well fed conditions, L4 animals cultivated at $20^{\circ} \mathrm{C}$ on nematode growth media with a thin layer of bacteria OP50 were single picked on the day before the experiment and stored at the conditioning temperature on a fresh plate with plenty of food (OP50). For starved conditions, the animals were moved into the fresh plate without food 2-4 $\mathrm{h}$ before the experiments at the conditioning temperature.

\section{Making a thermal gradient}

We used a thermal gradient device for the tracking experiment as described previously (Kuhara et al., 2011). Cooled water $\left(5-11^{\circ} \mathrm{C}\right)$ was maintained at a constant temperature using a bath recirculator AC150 (Thermo Fisher Scientific) and circulated through our custom thermal gradient device using a custom-built liquid pump (Tokai Hit). The thermal gradient device consisted of glass plates with indium tin oxide (ITO) connected to the liquid pump and a power supply, slidetrans RSA-5 (Rikosha). The ITO heats the glass plate according to the supplied electric current. A stable thermal gradient was established on a thermotaxis (TTX) assay plate containing $18 \mathrm{ml}$ of TTX medium with $2 \%$ agar in a $14 \times 10 \times 1.45 \mathrm{~cm}$ plastic dish (Eiken Chemical) within $>5$ min of being placed on the thermal device. The thermal gradient was measured at the beginning of every trial using both thermography with a TS9230W-A01 (NEC Avio) and a thermometer AM-8000K with a thermocouple probe (Anritsu). The agar medium on the plastic dish was cut into a $9 \times 6 \mathrm{~cm}$ rectangle, and $8 \mathrm{~m}$ glycerol was put on every edge of the cut agar to prevent the worms running away from the agar field.

\section{Tracking freely moving animals}

An ordinal analog video camera (CS230B; Olympus) captured freely moving animals at 30 frames per second (fps) with continuous halogen illumination using a $600 \mathrm{~nm}$ long-pass filter (R-66; Tokina). A motorized stage BioPrecision (Ludl) was controlled by the microscope-controlling software package $\mu$ Manager (Edelstein et al., 2010) with MATLAB (MathWorks). The MATLAB program identifies the head of the tracking worm in an acquired image based on the curvature of the tracking worm edge. Then, the program moves the stage according to the position of the identified head to keep the head in the center of the microscopic field and send trigger signals to the fluorescence imaging software described below (see Calcium imaging during tracking). The procedures of image acquisition to stage movement are executed every 0.5 s. $x-y$ coordinates are recorded with time stamps in each tracking trial. The time course of temperature experienced by the freely moving animal is calculated according to the recorded $x-y$ coordinates and the thermography, which taken at the beginning of each trial. We confirmed that the thermal gradient with our device kept a stable thermal gradient during the tracking trial.

\section{Calcium imaging during tracking}

We used a custom-built galvano-shutter system (Olympus Engineering) with a mercury lamp or SPECTRA light engine (Lumencor) for highspeed control of illumination. A $425 \mathrm{~nm}$ bandpass filter and $505 \mathrm{~nm}$ dichroic mirror (Olympus) were used for the galvano-shutter system; SPECTRA light engine included a bandpass filter (FF02-438/24-25; Semrock). A frost filter (Olympus) was used to evenly illuminate the field of view. We used a high fluorescence efficiency macro microscope (MVX10; Olympus) with 0.5 numerical aperture (NA) of $2 \times$ objective lens equipped with the thermal device and the motorized stage described above. A Dual-View equipped with $505 \mathrm{~nm}$ cube (Molecular Devices) 

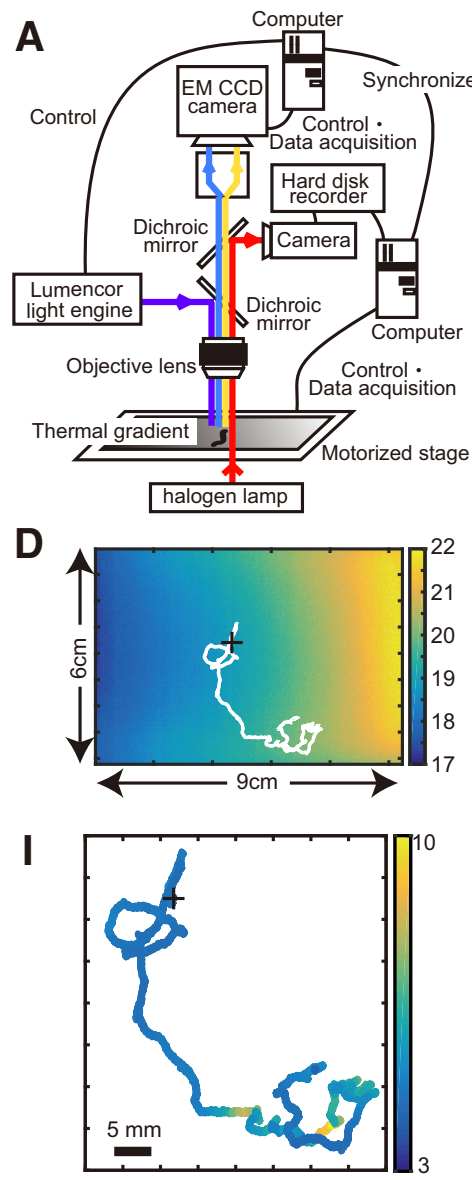

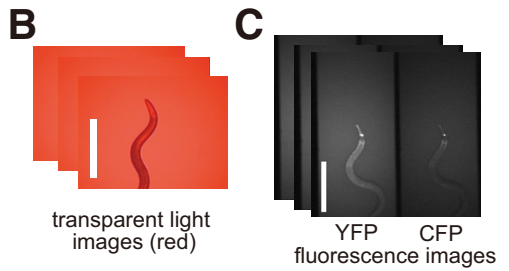

E
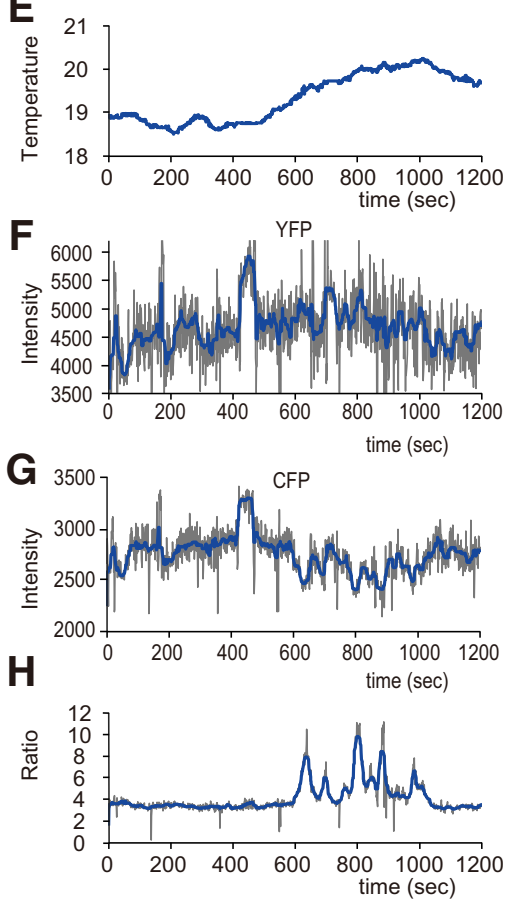

Figure 1. A tracking system for calcium imaging during thermotaxis. $A$, Schematic of the tracking microscope. Transparent red light images shown in $\boldsymbol{B}$ are processed in real time by the feedback controller for a motorized stage. A fluorescence illumination system and an EMCCD camera are controlled separately at lower frame rates to minimize photobleaching. These systems are synchronized with each other to prevent motion blur of fluorescent images. $C$, Fluorescence images were acquired using an EMCCD camera with a splitting channel into YFP and CFP. Scale bars: $\boldsymbol{B}, \boldsymbol{C}, 500 \mu \mathrm{m}$. D, A thermography image was taken in each experiment together with a calibrating temperature measurement using a thermocouple sensor. Projection of a wandering trajectory (white line) shows how the wandering worm searches thermal environment. + marks the starting point when recording starts. This worm was cultivated at $23^{\circ} \mathrm{C}$. , The time course of the temperature changes experienced by the migrating worm shown in $\boldsymbol{D}$. An estimation method for the temperature time course is described in Material and Methods. $\boldsymbol{F}$, Time courses of YFP intensity for the migrating worm shown in $\boldsymbol{D}$. G, Time courses of CFP intensity for the migrating worm shown in $\boldsymbol{D}$. $\boldsymbol{H}$, The time course of the YFP/CFP ratio of AFD neuron during thermotaxis shown in $\boldsymbol{D}$. $\boldsymbol{F}-\boldsymbol{H}$, The gray line denotes average AFD regional intensity (or ratio) in a frame (described in detail in Materials and Methods), and the blue line denotes the median for the time series of the average intensities (or ratio). Note that synchronized intensity changes of YFP and (FP were cancelled out in ratio $(\sim 420 \mathrm{~s})$. In contrast, reciprocal intensity changes in YFP and CFP fluorescence were reflected into high (or low) signals in ratio (after 600 s). $\boldsymbol{I}$, Mapped AFD activity of $\boldsymbol{H}$ onto the migratory coordinates. + marks the starting point. Scale bar, $5 \mathrm{~mm}$.

and EMCCD C9100-13 ImagEM (Hamamatsu Photonics) were used for acquiring yellow fluorescence protein (YFP) and cyan fluorescent protein (CFP) fluorescence images. Images were taken with $80 \mathrm{~ms}$ exposure time at $1 \mathrm{~s}$ intervals with $1 \times 1$ camera binning. Calcium imaging was regulated using MetaMorph (Universal Imaging).

The time course of YFP and CFP intensities showed a clear reciprocal relationship (Fig. $1 F, G$ ), indicating that the YFP/CFP represented the $\mathrm{Ca}^{2+}$ concentration change. Although unfocused blur sometimes caused synchronized intensity changes of YFP and CFP during the behavioral tracking, such unfavorable effects were cancelled out in YFP/CFP changes (Figs. $1 F, G$ ). We confirmed that the synchronized intensity changes were independent of temperature increases. In the case of a moving object, fluorescent emission signal is affected by the position in a microscopic field mainly because of inhomogeneous excitation light field. To prevent such positional artifacts, we monitored distribution of light intensity over the microscopic field for every experiment and calibrated the YFP and CFP signals.
Calcium imaging of fixed animals

The $20^{\circ} \mathrm{C}$ cultivated well fed animals were prepared as for the tracking experiments described above. Animals were fixed to an agar pad with polystyrene nanobeads and cover glass (Kim et al., 2013). The coverslip was placed on a Peltier-based thermo-controller (Tokai Hit) attached on an upright microscope (BX61WI; Olympus) with 0.9 NA of $40 \times$ objective lens. As in the tracking system, fluorescence was collected into a Dual-View with a $505 \mathrm{~nm}$ cube (Molecular Devices) and captured using an EMCCD C9100-13 ImagEM (Hamamatsu Photonics). Images were taken with $400 \mathrm{~ms}$ exposure at $1 \mathrm{~s}$ intervals with $1 \times 1$ binning. The temperature on the agar pad was monitored by using a thermometer system (DCM-20; Tokai Hit and Hamamatsu Photonics).

\section{Image analysis}

Background correction of non-uniform illumination. Fluorescence intensities of a moving object are likely to be inconsistent as a result of its position because illumination power is nonuniform over the microscopic field. As a consequence, the movement of a tracking animal may cause pseudo-activity during tracking. To correct the positional effect of illumination on neuronal fluorescence intensity, we monitored the positional profile of neuronal fluorescence intensity in a resting state (with constant temperature) and subtracted the estimated illumination effect from the tracking data. To estimate the illumination effect, we first imaged only the agar plate used for the tracking experiments. We then imaged neuronal intensities at the different positions of the microscopic field in a resting state after each tracking experiment: the worm used in a tracking experiment was glued onto an agar plate, and fluorescence images were taken by moving the stage to monitor neuronal intensities from various positions in the microscopic field. The plots of background intensity and resting neuronal intensity revealed an apparent linearity between the background intensity and neuronal intensity at a resting state (data not shown). Based on this relationship and the background intensity image, we estimated baseline neuronal intensity in any position and used this to cancel out the positional illumination effect in the tracking data. When we plotted the relationship between the YFP/CFP of the background agar intensity and YFP/CFP of the neuronal region, the plot showed that neuronal YFP/CFP linearly increased with the increase of background YFP/CFP. The steepness of the correlation slope was found to be dependent on the illumination/camera setting, whereas the relationship between the intensity of background and neuronal region showed positive correlation in any settings.

Analysis of calcium imaging for tracking animals. A neuronal region was defined according to the peak intensity and size ( 9 pixels) in a YFP image; a neuronal region for a CFP image was defined according to the corresponding YFP image because of the dimmer CFP images. Then, the average fluorescence intensities of the neuronal regions were calculated for each image. Intercellular calcium concentration change was estimated by taking the acceptor/donor (YFP/CFP) fluorescence ratio. A median filter was applied to the time course ratio to eliminate the noise independent from calcium signal. For the analysis of temperature dynamics evoking AFD intermittent activities (see Figs. $2 A, 6 E$ ), we defined a peak delta 

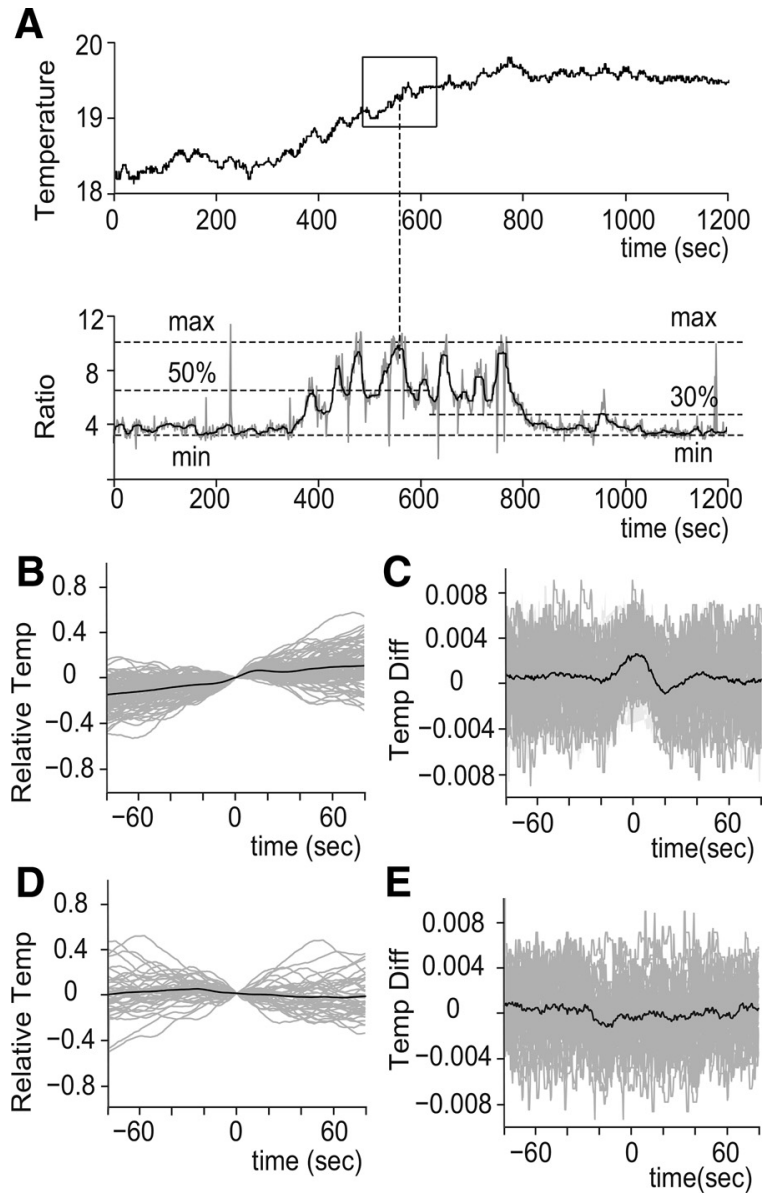

Figure 2. Temperature dynamics that evoke AFD intermittent activities. $\boldsymbol{A}$, An example of the extracted temperature experience related to AFD activity. An intermittent activity is defined by a peak and threshold (left side, $50 \%$, at the bottom). Temperature time courses corresponding to peaks were extracted inside time windows. An example is shown in the black square in the top. The gray line in the bottom denotes raw signal, and black denotes the median filtered signal. A threshold for a flat region (30\%) is shown at the right side of the bottom. $\boldsymbol{B}$, Extracted temperature time courses are drawn relative to the AFD activity peak (horizontal, the peak is time $=0$ ) and the temperature relative to the temperature at the peak (vertical; $0^{\circ}($ at time 0 ). Note that time 0 of the average shows clear increase of temperature. $\boldsymbol{C}$, The differential of $\boldsymbol{B}$. For $B$ and $C, 98$ AFD activity peaks were extracted from 10 fed worms cultivated in $20^{\circ}$. D , Extracted temperature associated with flat regions plotted over time. A flat region was defined as a sequence of activity under $30 \%$ threshold longer than $15 \mathrm{~s}$. Forty-two periods were extracted from the same dataset used in $\boldsymbol{B}$ and $\boldsymbol{C}$. $\boldsymbol{E}$, The differential of $\boldsymbol{D}$. $\boldsymbol{B}-\boldsymbol{E}$, Gray shows each extracted time course, and black shows the average.

YFP/CFP that was larger than a 50\% threshold of maximum delta YFP/ $\mathrm{CFP}$ in a trial as an AFD phasic activity. The image processing program for the tracking data was written in MATLAB (MathWorks).

Analysis of calcium imaging for fixed animals. We analyzed the calcium imaging data of fixed animals using MetaMorph (Molecular Devices). Background subtraction was executed using the built-in function called statistical correction. A neuronal region was defined with a threshold in a YFP image determined by an observer. To prevent unexpected motion artifacts, positional correction of the defined neuron was executed with the center of mass of the neuronal region. Then, average fluorescence intensities in the neuronal region were calculated for each image. Intercellular calcium concentration change was estimated by taking the change of acceptor/donor (YFP/CFP) fluorescence ratio ( $\Delta$ ratio).

\section{Estimation for fully sampled response functions}

The response of AFD neuron at time $t, y(t)$, was modeled by linear convolution of input $x(t)$ with the response function $w(t)$ :

$$
y(t)=w_{0}+\int_{0}^{t} w(t-\tau) x(\tau) d \tau+\varepsilon,
$$

where $w_{o}, w(t), x(t)$, and $\varepsilon$ indicate basal activity of the AFD neuron, the response function, temperature input, and noise, respectively. The response function $w(t)$ represents the impulse response, which corresponds to evoked activity of AFD in response to impulse inputs of $x$ : $x(t)=\delta(t)$, where $\delta(t)$ denotes Dirac's delta function.

To estimate the response function, we performed the ridge regression, in which the parameter set $W=\left\{w_{o}, w_{1}, w_{2}, \ldots, w_{r}\right\}$ was in total called a response function and determined by minimizing the objective function consisting of the sum-of-squares error and a regularization term:

$$
\frac{1}{2} \sum_{t=r+1}^{N}\left\{v_{t}-\left(w_{0}+\sum_{\tau=0}^{r} w_{\tau} u_{t-\tau}\right)\right\}^{2}+\lambda\|W\|^{2}
$$

where $N$ and $\lambda$ indicate the total number of sampling points in observed time series data and regularization parameter, respectively. $r$ denotes the maximum delay time, or time window. $u$ and $v$ are temperature input and calcium activity, respectively. Note that the introduction of regularization avoids overfitting and improves generalization.

\section{Reduced parameter estimation of the response function}

In terms of Laguerre orthogonal polynomials, generally, the response function $K(t)$ can be written in the following form: $K(t)=$ $e^{-\lambda t}\left(\Sigma_{i} \alpha_{i} L_{i}(t)\right)$. According to the fully sampled response functions of the AFD neuron, three parameters $\left\{\lambda, \alpha_{0}, \alpha_{1}\right\}$ of impulse response $K(t)=e^{-\lambda t}\left(\alpha_{0}-\lambda \alpha_{1} t\right)$ are sufficient to describe the AFD response in our experimental conditions, similar to the study of bacterial chemotactic response (Celani and Vergassola, 2010; Masson et al., 2012). The Nelder-Mead simplex method was used to determine these parameters (Lagarias et al., 1998).

\section{Nonlinear transformation}

The Hill function, $H(t)$, was incorporated into the response model to represent the operating range of AFD to temperature. Based on the observation of AFD activity related to the operating range, we defined the Hill function as follows:

$$
H(t)=\frac{(t-T s)^{n}}{K d+(t-T s)^{n}} \text { when } t>T s, H(t)=0 \text { when } t<T s
$$

where $t, T s, K d$, and $n$ denotes temperature, cultivated temperature, dissociation constant, and Hill coefficient, respectively. We used the parameters $K d=8$ and $n=2$ according to the observed responding range of AFD of $20^{\circ} \mathrm{C}$ cultivated animals.

\section{Estimation for thermal environment}

The thermal environment, $\tilde{T}(x, y)$, was modeled as a second-order linear polynomial:

$$
\tilde{T}(x, y)=\sum_{i, j=0}^{i+j \leq 2} a_{i j} x^{i} y^{j}
$$

with the assumption that temperature smoothly changes along the $x-y$ plane in our experimental condition. Each parameter $a_{i j}$ was determined by least-squares fitting with minimizing the following objective function:

$$
\frac{1}{2} \sum_{t}(\operatorname{deconv}(v, w(t))-\tilde{T})^{2}
$$

where temperature change deconv $(v, w(t))$ was calculated by the deconvolution of the time course of AFD activity $v$ with the estimated response function $w(t)$. We used the Wiener filter for deconvolution algorithm without noise term.

\section{Evaluation for the reconstruction}

The reconstructed AFD activity from temperature input and the identified response function was evaluated by variance accounted for (VAF), defined as follows: 
A

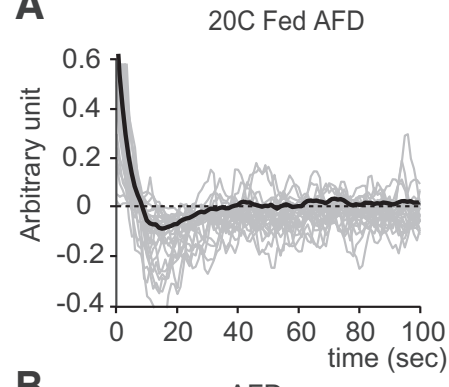

B
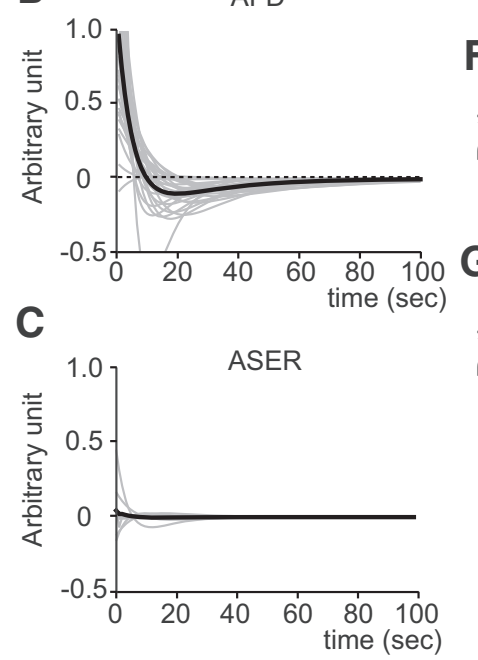

D

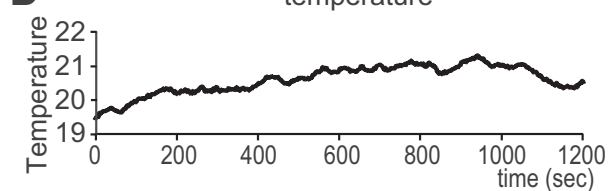

E

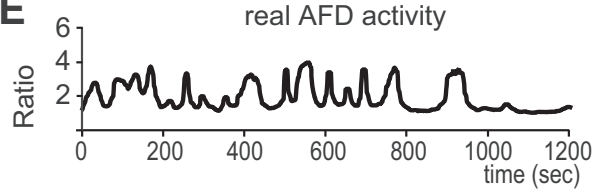

$\mathbf{F}$
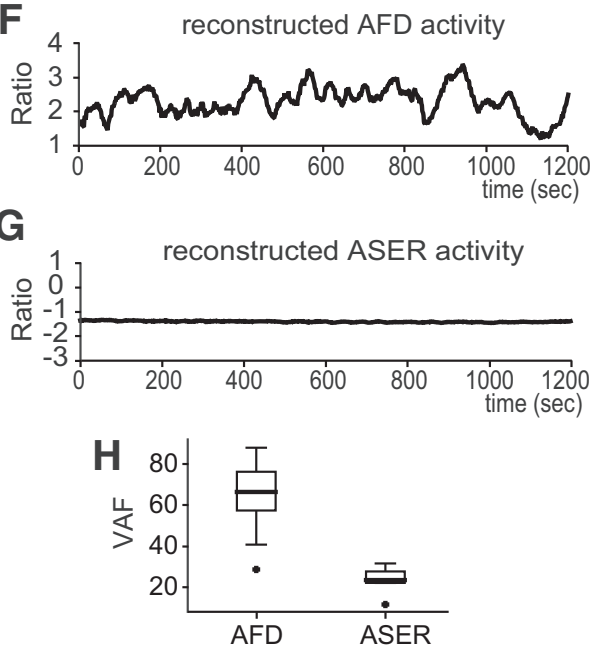

Figure 3. Estimation of the response function of AFD and reconstruction of AFD activity. $A$, Fully sampled response functions of AFD thermosensory neuron in response to thermal stimulus $\left(20^{\circ} \mathrm{C}\right.$ cultivated well fed animals, $\left.n=27\right)$. B, AFD response functions with the three-parameter model: $K(t)=e^{-\lambda t}\left(\alpha_{0}-\lambda \alpha_{1} t\right)$. The same dataset in $A$ is used $\left(20^{\circ} \mathrm{C}\right.$ cultivated well-fed animals, $n=$ 27). $C$, The response functions of ASER NaCl sensory neuron in response to thermal stimulus with the three-parameter model $\left(20^{\circ} \mathrm{C}\right.$ cultivated, well fed, $n=9)$. $\boldsymbol{A}-\boldsymbol{C}$, The gray lines denote individual estimations, and the black line denotes their average. $\boldsymbol{D}, \mathrm{A}$ representative temperature time course of a freely moving animal during thermotaxis. $E$, The observed real AFD activity in response to the temperature input shown in $\boldsymbol{D}$. $\boldsymbol{F}$, Reconstructed AFD neural activity from the estimated average response function shown as the black line in $\boldsymbol{B}$ and temperature time course shown in $\boldsymbol{D}$. $\boldsymbol{G}$, Reconstructed ASER activity from the average response function shown as the black line in $\mathbf{C}$ and the temperature input shown in $\boldsymbol{D}$. $\boldsymbol{H}$, Performance of reconstructed neural activities in VAF (see Materials and Methods).

$$
\mathrm{VAF}=100 \% *\left(1-\frac{\operatorname{var}(y-\hat{y})}{\operatorname{var}(y)}\right),
$$

in which $y$ denotes monitored AFD activity and $\hat{y}$ denotes estimated AFD activity by the response function and temperature input. VAF indicates the percentage of predictive power from the model (Kato et al., 2014).

To evaluate similarity for the reconstructed thermal environment, we used the structural similarity (SSIM) index (Wang et al., 2004):

$$
\begin{aligned}
S(x, y)= & l(x, y)^{\alpha} \cdot c(x, y)^{\beta} \cdot s(x, y)^{\gamma} \\
& =\left(\frac{2 \mu_{x} \mu_{y}+\mathrm{C}_{1}}{\mu_{x}^{2}+\mu_{y}^{2}+\mathrm{C}_{1}}\right)^{\alpha} \cdot\left(\frac{2 \sigma_{x} \sigma_{y}+\mathrm{C}_{2}}{\sigma_{x}^{2}+\sigma_{y}^{2}+\mathrm{C}_{2}}\right)^{\beta} \cdot\left(\frac{\sigma_{x y}+\mathrm{C}_{3}}{\sigma_{x} \sigma_{y}+\mathrm{C}_{3}}\right)^{\gamma}
\end{aligned}
$$

where $\mu_{x}$ and $\mu_{y}$ are the local sample means of $x$ and $y$, respectively, $\sigma_{x}$ and $\sigma_{y}$ are the local sample SDs of $x$ and $y$, respectively, and $\sigma_{x y}$ is the local sample cross-correlations of $x$ and $y . \mathrm{C}_{1}, \mathrm{C}_{2}$, and $\mathrm{C}_{3}$ are all regularization constants to stabilize each terms (we used $\mathrm{C}_{1}=0.01^{2}, \mathrm{C}_{2}=0.03^{2}$, and $\mathrm{C}_{3}$ $=\mathrm{C}_{2} / 2$ ). In short, the SSIM index compares three elements (luminance, contrast, and correlation) between two images. $\alpha, \beta$, and $\gamma$ are exponents for balancing each element. We used $\alpha=0, \beta=1$, and $\gamma=1$ because the reconstruction of temperature from fluorescence signal does not ensure absolute value and luminance highly depends on the expression level of the fluorescence probe. We used the MATLAB built-in function of the SSIM index.

\section{Results}

Calcium imaging of AFD activity in freely moving $C$. elegans

To address how AFD neurons encode the thermal environment, we developed an automated system that allows simultaneous monitoring of $\mathrm{Ca}^{2+}$ signal from neurons as fluorescence images and the behavioral trajectory of freely moving animals as $x-y$ coordinates. We used the ratiometric calcium probe yellow cameleon (YC3.60) to avoid movement artifacts and expressed it specifically in AFD neurons using the $g c y-8$ promoter (see Materials and Methods). A tracking system with a visual feedback controller (Fig. 1A) allowed us to track the head of the moving animal using images acquired by transillumination with red light (Fig. 1B), while simultaneously acquiring fluorescence images (Fig. 1C), the two-dimensional migration trajectory (Fig. 1D), and thermography of the animal's environmental field (Fig. 1D). The fluorescence images were acquired independently from the frequently acquired transparent red light images (30 fps) to reduce photobleaching of the fluorescence probe. By combining these data, we could capture both the temperature and the AFD activity of a freely moving animal on a thermal gradient over time (Figs. $1 E, H$ ).

We found that the AFD neuron showed an intermittent phasic increase of calcium in response to slight increases in temperature (Fig. $1 E, H$ ). Although previous studies reported that the AFD neurons show short-term adaptation in response to step-like temperature increases (Kimura et al., 2004) and sinusoidal temperature input induced the phase-locked activity of AFD around cultivation temperature (Clark et al., 2006), our present results showed that adaptation of AFD gave rise to intermittent $\mathrm{Ca}^{2+}$ activity in response to a shallow temperature $\operatorname{ramp}\left(0.1^{\circ} \mathrm{C} / \mathrm{min}\right)$ in animals moving freely on a thermal gradient (Fig. 1E,H). Furthermore, we found that the duration of the spike-like activities of AFD was $\sim 20 \mathrm{~s}$, and this was insensitive to the quicker (timescale of seconds) increase of temperature. These intermittent neural activities suggest that the representation of the continuous shallow temperature gradient in the dynamics of AFD activity is in the form of discrete stereotypical peaks.

\section{Temperature increases trigger phasic AFD activity}

To reveal the environmental stimuli that triggered the intermittent phasic activity of AFD neurons, we analyzed the detail of the relationship between the thermal stimulus and the activity of AFD neurons in freely behaving animals. We extracted the temperature profile in a time window of $80 \mathrm{~s}$ around the defined AFD phasic activity (Fig. 2A; see Materials and Methods). Then, averaging of the temperature profile was triggered on peaks in AFD activity. These profiles showed clear correlation between the de- 
fined AFD phasic activity and temperature increase (Fig. 2B, time 0 ).

Calculation of the temperature differential further revealed that the timing of phasic activity was associated with an increase and a subsequent slight decrease of temperature (Fig. 2C). The duration of the temperature increase before the appearance of the phasic AFD activity was $\sim 20 \mathrm{~s}$. The increase continued for $10 \mathrm{~s}$ after the peak of AFD phasic activity, and then a small temperature decrease appeared $10 \mathrm{~s}$ after the AFD phasic activity and reached the lowest value within $20 \mathrm{~s}$ after the peak phasic activity. These results suggested that most of the intermittent activities of AFD were followed by the decrease of temperature. To examine this further, we defined silent periods as intervals longer than $15 \mathrm{~s}$ under $30 \%$ of threshold and extracted their respective temperature to compare with the AFDpeak-triggered average of the temperature. The average of the corresponding temperature sequences and its differentials (Figs. 2D,E) showed no increase of temperature. Mapping of AFD activity onto the navigation trajectory revealed that the turning frequency was increased around peak AFD activities (Fig. 1I, right bottom region). Because the increase in temperature evokes frequent turnings of worms (Ryu and Samuel, 2002), we hypothesized that temperature increases evoke the phasic AFD activity, which is associated with turning behaviors; after the phasic AFD activity, the evoked turnings result in the animals experiencing a decrease in temperature if the animals are on a thermal gradient.

\section{Estimation of an AFD response function and reconstruction of AFD activity}

To elucidate how the temperature stimulus is processed by AFD neurons of freely moving animals, we estimated the response function of AFD, which enables to characterize the relationship between the input and output of the AFD neuron as a temporal filter. A fully sampled linear function was estimated by least-square regression (Fig. 3A). The estimated response functions of freely moving animals on a thermal gradient showed a typical shape of sensory impulse response, which corresponds to the observed phasic activities. Then, we modeled the impulse response as an exponential function with three parameters, similar to the bacterial chemotactic response (Fig. 3B; Celani and Vergassola, 2010). The shape of the response functions suggested that AFD detects the differential of temperatures during continuous temperature input: a fast positive response followed by a slow negative response is indicative of detection of temperature increase and adaptation to the stimulus. We propose that this response property of AFD neurons contributes to the behavioral responses of animals to temperature change, in particular in controlling turning frequency change, extending previous work (Ryu and
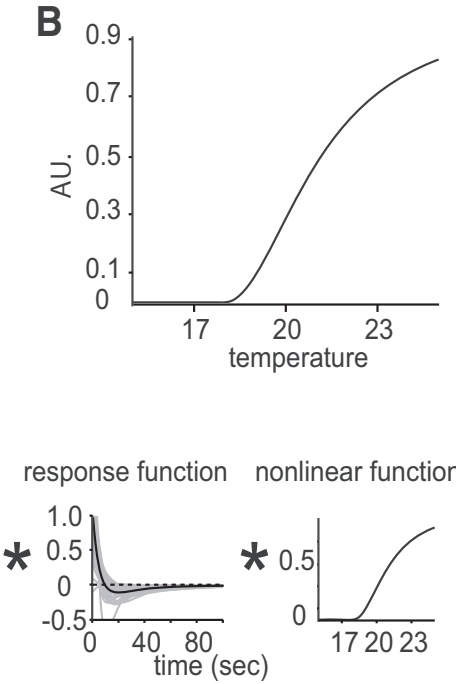

thermal stimulus
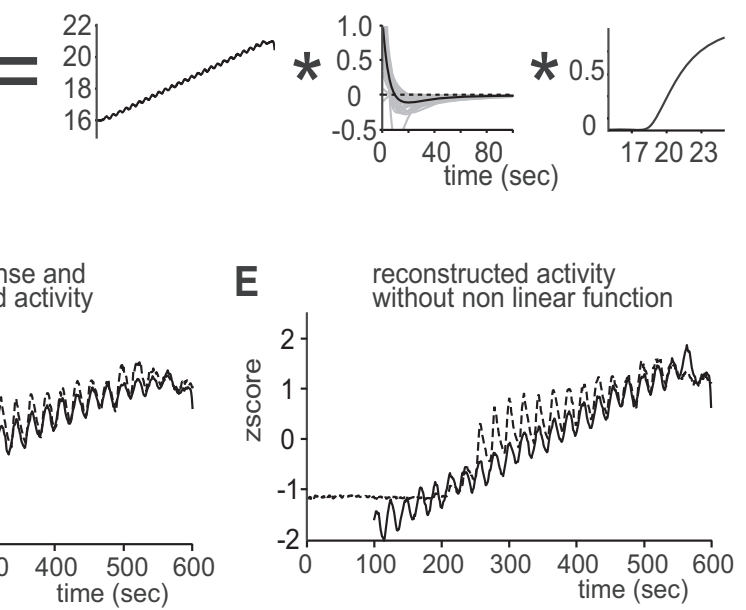

Figure 4. An AFD response model including the operating range parameter (the $L-N$ model; see Results). $\boldsymbol{A}$, Sinusoidal thermal input (top) and corresponding AFD response (bottom, gray lines for individuals and black line for the average; $20^{\circ} \mathrm{C}$ cultivated, well-fed, $n=10$ ). AFD starts to respond above cultivation temperature $-2^{\circ} \mathrm{C}$. $\boldsymbol{B}$, A nonlinear function in terms of the Hill equation

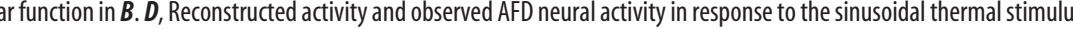
hown in the top of $\boldsymbol{A}$. The dotted line denotes observed AFD activity and the solid line for the reconstruction. $\boldsymbol{E}$, Reconstruction without the nonlinear function failed to reproduce the operating range of AFD activity. The dotted line denotes observed AFD activity, and the solid line denotes the reconstruction.

Samuel, 2002; Zariwala et al., 2003). We think the identified temporal AFD response represents a key component for constructing the behavioral regulatory signal.

To explore this further, we simulated AFD activity on a computer from a recorded temperature input (Fig. 3D) using the estimated average response function (Fig. 3B). Simulated AFD activity reproduced a similar time course as experimentally obtained AFD activity (Fig. $3 E, F$ ). As a control experiment, we estimated the response function of ASER for temperature input. ASER is a salt-sensing gustatory neuron (Suzuki et al., 2008), not known to have any thermosensory properties. We constructed a strain expressing cameleon (YC3.60) in ASER by using the $g c y-5$ ASER-specific promoter and confirmed clear responses to $\mathrm{NaCl}$ concentration change. The estimated response function of this gustatory neuron did not show any characteristic response property to temperature input (Fig. 3C). Comparison of VAF, which is a performance index for reconstructed neural activities, demonstrated superior fitness of the estimated AFD response function to that of ASER (Fig. $3 H$ ). Also, there was no clear relationship between temperature input and the ASER activity (data not shown); the reconstructed activity of ASER represented a noninformational property (Fig. $3 G$ ). These results control for the 

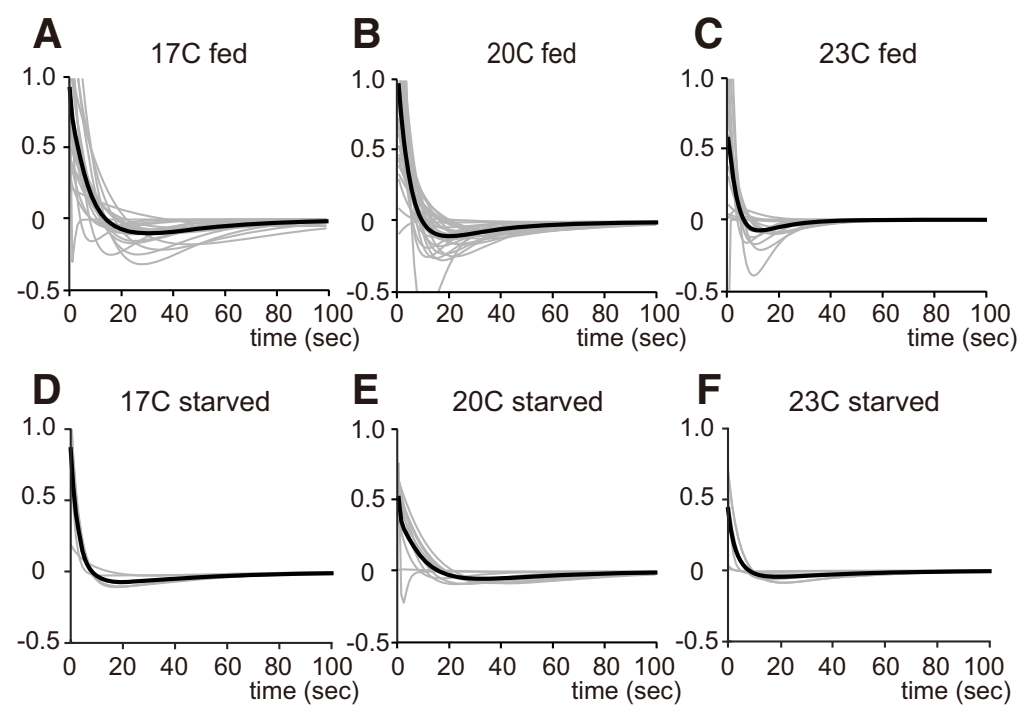

G

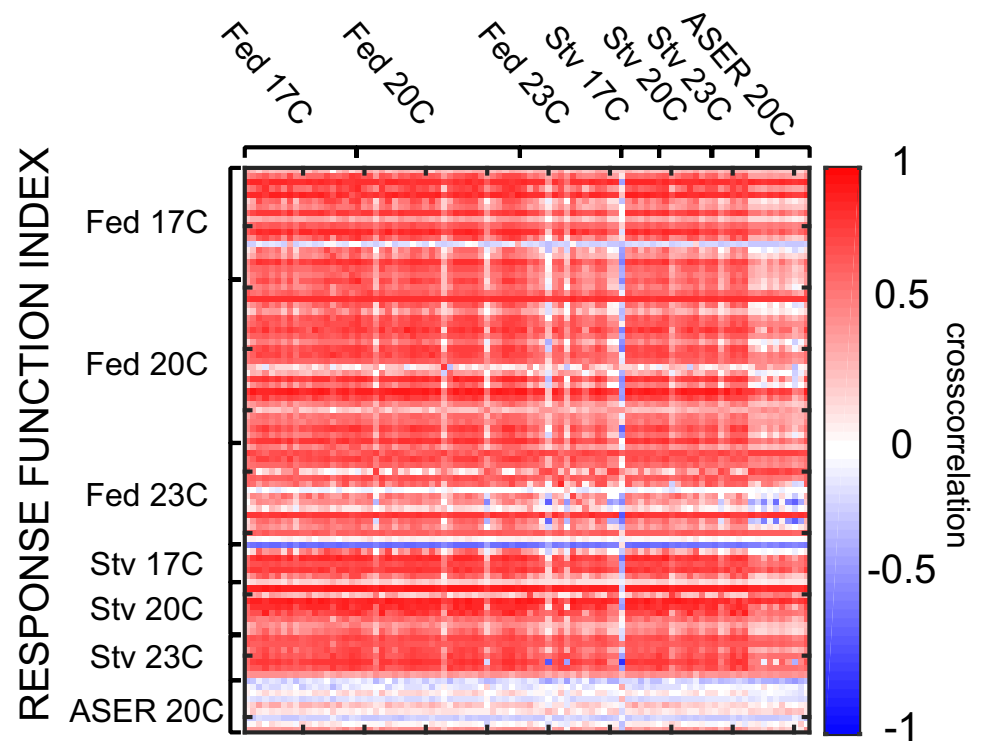

Figure 5. Conserved property of AFD response function. $A-F$, Response functions at different conditions. The gray lines fare or individual estimation, and the black lines are for the averages. $\boldsymbol{A}, \mathrm{A} 17^{\circ} \mathrm{C}$ cultivated well fed worm $(n=19) \cdot \boldsymbol{B}, \mathrm{A} 20^{\circ} \mathrm{C}$ cultivated well fed worm; same data as Figure $3 B(n=27) . \boldsymbol{C}, A 23^{\circ} \mathrm{C}$ cultivated well fed worm $(n=14) . \boldsymbol{D}, A 17^{\circ} \mathrm{C}$ cultivated starved worm $(n=5) . \boldsymbol{E}, \mathrm{A} 20^{\circ} \mathrm{C}$ cultivated starved worm $(n=9) \cdot \boldsymbol{F}, \mathrm{A} 23^{\circ} \mathrm{C}$ cultivated starved worm $(n=7)$. G, Validation of the reconstruction with different combinations of experimental conditions. The heat map shows cross-correlation coefficients between the reconstructed activity with the response function estimated with labeled condition (raw) and the observed activity in the labeled condition (column). Except for the ASER experiments, most of the combination shows high correlation (Fed 17C, $n=19$; Fed 20C, $n=27$; Fed 23C, $n=16$; Stv 17C, $n=5 ;$ Stv 20C, $n=9$; Stv 23C, $n=7$; Fed ASER 20C, $n=9$ ).

possible artifacts that could have arisen during the acquisition or the analysis of calcium imaging data from freely moving animals.

\section{The operating range of AFD responses and nonlinear transformation}

Animals exposed to sinusoidal temperature oscillations showed clear phase-locked responses around the cultivation temperature (Fig. 4A) as reported previously (Clark et al., 2006), which is likely attributable to the cultivation temperature-dependent operating range of AFD responses. Because freely behaving animals tended to migrate toward their cultivation temperature on a thermal gradient, our sampling of freely moving animals while estimating AFD response properties were probably biased in favor of the operating range. Therefore, we incorporated the mechanism of the operating range into our AFD response model, by deriving a linear-nonlinear $(\mathrm{L}-\mathrm{N})$ model that consists of the obtained response function and a nonlinear transformation representing the operating range. Based on the experimental considerations and assumptions of allosteric molecular signaling (Hogg et al., 2005), we used the Hill equation to express the nonlinear transformation (Fig. 4B). According to the L-N model, the external stimulus was filtered by the response function, which was estimated from freely moving animals, and then transformed by the nonlinear Hill equation (Fig. 4C). Computer-simulated AFD activity for a defined sinusoidal temperature stimulus matched the real AFD activity (Fig. 4D). The reconstruction without the Hill transformation lacked the proper operating range, although it kept the phase-locked oscillation in response to the sinusoidal thermal stimulus (Fig. 4E). Thus, we confirmed that our $\mathrm{L}-\mathrm{N}$ model separately implements the response property of AFD by the estimated response function and the operating range of AFD by the nonlinear transformation, respectively.

\section{The response function of AFD is independent of different conditions of food and cultivation temperature}

Because thermotaxis behavior is known to be modulated by cultivation temperature and feeding state (Hedgecock and Russell, 1975; Mohri et al., 2005; Kodama et al., 2006), we investigated the response patterns of AFD when we changed the animal's cultivation temperature or food condition. The shapes of response functions kept an initial positive response and a delayed negative response, regardless of cultivation temperatures (Fig. 5A-C). Although our estimation was derived mostly from the animals behaving within the operating ranges, because of the animals tendency to migrate around their cultivation temperature, the deduced response functions suggest that the response property of AFD is consistent over several cultivation temperature conditions.

Similarly, we found that starved animals also kept the initial positive and delayed negative response properties of AFD (Fig. $5 D-F)$. Given that food condition is known to affect thermotaxis, the invariable AFD responses implicate that the relationship between AFD activity and behavioral components is different between different food conditions. As we have shown with the $\mathrm{Ca}^{2+}$ imaging data during thermotaxis, the phasic activities of AFD are correlated imperfectly with the turning behaviors during migration on the thermal gradient in a fed condition (Figs. 1I, 6F). Although the timing of calcium peaks in AFD neurons was not correlated to turns by a one-to-one correlation, peak AFD activ- 
ities were accompanied frequently by turns. Such loose coupling between AFD activity and a turn collapsed under starved conditions (Figs. 6A-E, G). These data suggested that the sensory signal of AFD is conveyed to a behavioral regulatory signal in fed conditions but not in starved conditions.

To evaluate the compatibility of the estimated response functions in different conditions, we calculated the cross-correlation between the experimentally observed AFD activity and the reconstructed AFD activity by the response functions derived from animals cultivated under different conditions. The cross-correlations of the different combinations of conditions are summarized in Figure $5 G$. The high cross-correlation coefficients in most of combinations in Figure $5 G$ suggested compatibility of each estimated response function, which is in accordance with an invariant shape of the estimated response functions. In contrast to AFD, ASER showed no correlations in any combinations. Thus, our response model well explained the AFD neuronal activity in response to thermal stimulus during thermotaxis, and invariable response property of AFD was compatible within different conditions.

\section{Reconstruction of the temperature environment}

We found that the response functions of AFD to thermal input were unvarying among different individuals. Moreover, reconstructed AFD activities by convoluting the response functions and temperature inputs were similar to the observed corresponding AFD activities. These results suggest that, once we have identified the response function, temperature inputs can be estimated by deconvolving AFD activities over time. In addition, migration coordinates and the estimated temperature at each point should have the information needed to establish the thermal environment without direct measurement of temperatures. Based on this hypothesis, we tried to estimate the temperature environment using AFD activity, trajectories, and the response function of the freely behaving animals. First, the AFD activity of a well fed animal on a thermal gradient (Fig. 7A) was deconvolved with the identified response function to estimate temperature input along the migration trajectory. Then, we reconstructed the relative thermal landscape of a moving animal based on the deconvolved temperature and observed migration trajectory, by fitting to a polynomial model of the thermal plane (Fig. $7 B$; see Materials and Methods). The reconstructed temperature environment was almost identical to the observed temperature environment (Fig. 7 B,C). We eliminated possible artifacts caused by data acquisition or the modeling procedure itself: the reconstructed thermal environment from ASER activity (Fig. 7D) collapsed the environmental linear thermal gradient (Fig. $7 E, F$ ). The distribution of the cross-correlations between reconstructed thermal environments for starved).

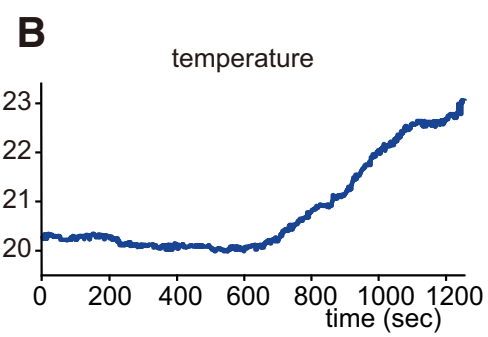

D
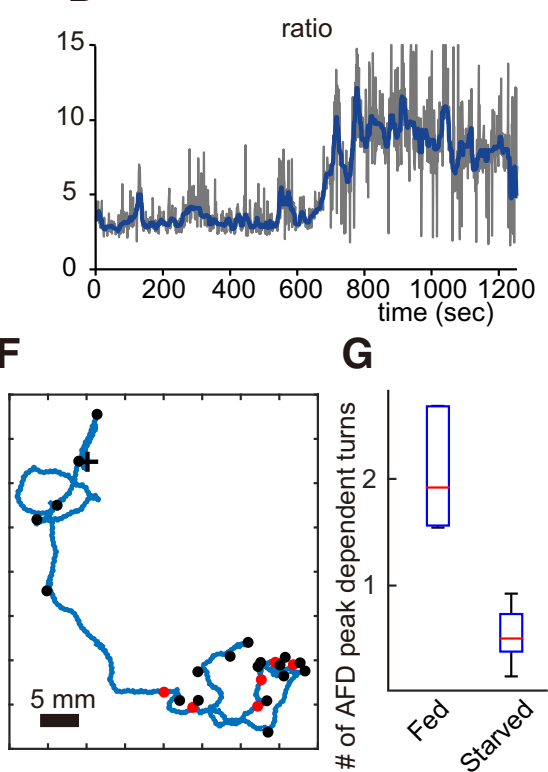

Figure 6. AFD activity of a starved animal during thermotaxis. $\boldsymbol{A}$, Thermography and migration trajectory of $20^{\circ} \mathrm{C}$ cultivated correlation to directional turning different from fed animals. $\boldsymbol{D}$, The time course of AFD activity in the starved conditioned animal sote that AFD starts to respond to temperature increases at $\sim 800 \mathrm{~s}$, similar to the fed condition. $\boldsymbol{E}, \boldsymbol{F}$, Correlation . + denotes the starting points in $\boldsymbol{A}, \boldsymbol{C}, \boldsymbol{E}$, and $\boldsymbol{F}$. G, Frequency of activity peak-dependent turn in fed and starved animals cultivated in $20^{\circ} \mathrm{C}$. The turns within $30 \mathrm{~s}$ of an AFD peak were counted, and the frequency was calculated by (the number of turns)/(the number of AFD peaks) (total of 67 peaks and 142 turns of five animals for fed, 44 peaks and 25 turns of five animals

and corresponding observed thermal landscapes showed high reproducibility of the reconstructed thermal environments from AFD activity (Fig. $7 G$ ). This correlation is kept in the AFD activity-based reconstruction with different cultivation temperatures or food conditions but is not kept in the ASER activity-based reconstruction or the random activity-based reconstruction. Similar to crosscorrelation, the SSIM index (Wang et al., 2004) showed a high performance of the thermal environmental reconstruction based on the AFD response function (Fig. $7 H$ ). These results showed that the identified response function and a series of AFD activity along the migration trajectory include the information required to predict (or "decode") the temperature environment.

\section{Discussion}

Quantitative analysis and mathematical description of functional components provide frameworks to understand the dynamic nature of neural circuits. To understand the encoding mechanisms 


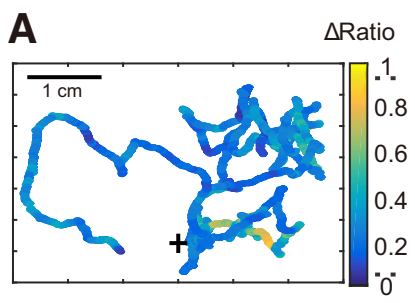

D

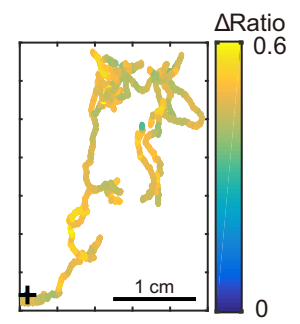

G Crosscorrelation

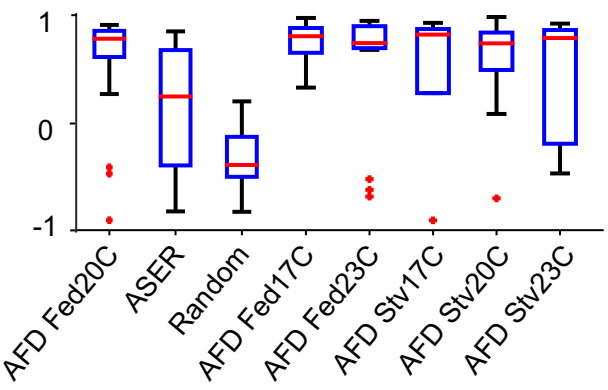

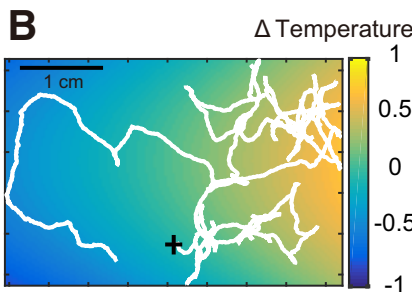

E

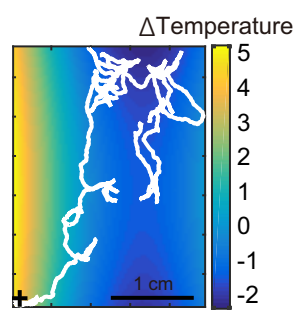

H

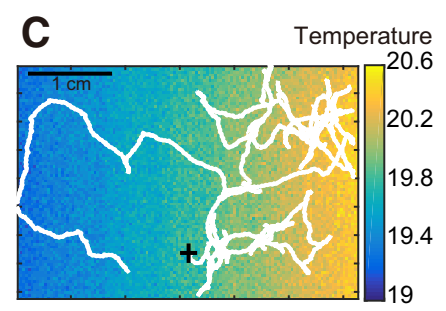

$\mathbf{F}$

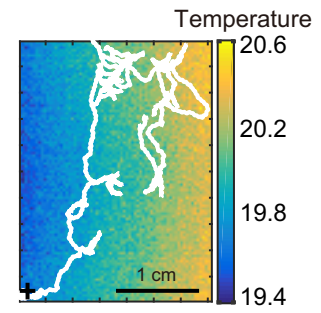

SSIM

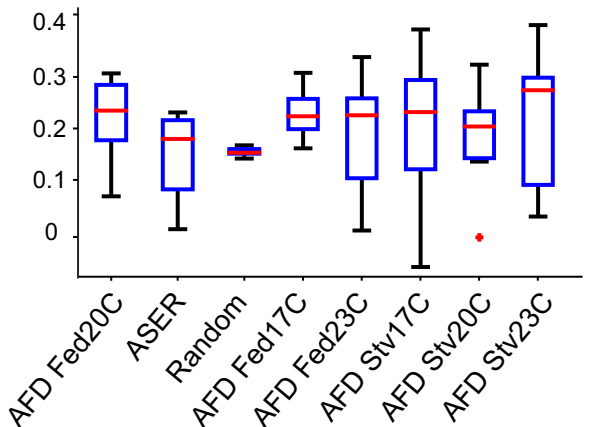

Figure 7. Reconstructed thermal environment based on the time course of AFD activity and wandering trajectories. A, AFD activity mapped on the migration trajectory. Twenty-five minutes of tracking for a $20^{\circ} \mathrm{C}$ cultivated fed worm. $\boldsymbol{B}$, Reconstructed temperature environment from deconvolution of AFD activity and wandering trajectory shown in $\boldsymbol{A}$. C, The observed wandering trajectory and thermography image of the plate corresponding to the animal in $\boldsymbol{A}$ and $\boldsymbol{B}$. D, ASER activity mapped on the migration trajectory. Twenty-five minutes of tracking for a $20^{\circ} \mathrm{C}$ cultivated fed worm. $\boldsymbol{E}$, Reconstructed temperature environment with the average response function of ASER shown in Figure 3C, ASER activity, and the wandering trajectory shown in $\boldsymbol{D}$. $\boldsymbol{F}$, The observed migration trajectory and thermography image of the plate corresponding to $\boldsymbol{D}$ and $\boldsymbol{E}$. $\boldsymbol{G}$. Cross-correlation between acquired thermography and thermal environmental reconstruction from AFD in different conditions, ASER, and random activity with wandering trajectory. $\boldsymbol{H}$, The SSIM index (see Materials and Methods) between acquired thermography and thermal environmental reconstruction from

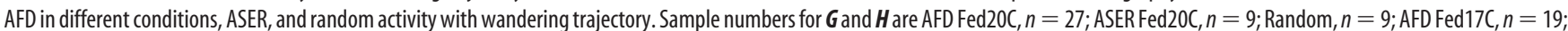
AFD Fed 23C, $n=16 ;$ AFD Stv17C, $n=5 ;$ AFD Stv20C, $n=9 ; \operatorname{AFD~Stv23C,~} n=7$.

of environmental temperature by sensory neurons, we investigated freely moving animals on a thermal gradient and quantified the response property of the thermosensory neuron AFD. The estimated response functions were invariant among different cultivation conditions, and the shape of the response function indicates adaptation and differential detection of input signal based on a time window of $20 \mathrm{~s}$, a timescale comparable with that of behavioral components used by the animal to navigate, such as turning. We incorporated a nonlinear function into the AFD response model to reflect the dependence of the AFD operating range on cultivation temperature (Kimura et al., 2004; Clark et al., 2006). Reconstruction of AFD activity using the estimated response function and the nonlinear property of the operating range recapitulated the real AFD activity in response to temperature input. Conversely, we were able to reconstruct the thermal environment from AFD activities over the course of navigation. Thus, AFD activities of the behaving animals contain enough information to reproduce the thermal environment.

\section{Encoding of thermal environment by AFD neuron}

AFD neurons of freely moving well fed animals on a shallow thermal gradient showed intermittent calcium responses. This observation suggests a discrete encoding system of AFD neurons even on a continuous thermal gradient. Although the response property of AFD contributes to the generation of pulse-like activities, regulation of changes in input pattern seems to influence the generation of these intermittent pulses. In fact, directional turnings of a fed animal seemed to correlate with intermittent pulse-like activities (Figs. 1I, $6 F$ ), whereas a starved animal showed straightforward movements with gradual AFD activities, which seem to be caused by summations of pulse-like activities (Fig. $6 A-D$ ). We assume that directional turning on a thermal gradient causes a change in the temperature input experienced by the animal, and, consequently, the perturbation contributes to generate pulse-like AFD activities of freely moving animals. The differential of the phase response curve of the AFD neuron showed a temperature decrease at $20 \mathrm{~s}$ after the timing of phasic activities (Fig. 2C), implying that turnings evoked by the AFD pulse might cause this temperature decrease. Together, the behavioral feedback on the thermosensory input may play a role to recognize environmental information in a freely moving condition. The discrete encoding system of an AFD thermosensory neuron, as a consequence of the behavioral feedback, would help animals navigate a continuous thermal environment. Put differently, the behavioral feedback may amplify the thermosensory 
signal of AFD by making an increase of AFD activity into a distinct phasic activity.

We successfully reconstructed both AFD activity and the thermal environment by estimating the response function, suggesting that the activity of AFD encodes differential information of the temperature sequence experienced by animals. However, because we reconstructed only a relative temperature time course, the mechanism of encoding absolute temperatures still remains to be clarified. The encoding of absolute temperatures may relate to the mechanism of temperature memory, because the operating range of AFD depends on the absolute temperature to which animals were exposed previously. Fluorescence-based imaging of neural activity may not be suitable for monitoring absolute temperatures, which are coded in the AFD neurons, because fluorescence intensity varies depending on the expression of probes, making it hard to compare across different individuals. New assays may be required to address questions related to the mechanism underlying the sensation of absolute temperature.

\section{Behavioral regulation according to the encoded signal}

We observed that the spike-like AFD activity did not directly reflect the behavioral output, although the frequency of directional turnings seemed to increase with high AFD activity in the fed condition. Although the sensory neurons keep the linearity of their response to the environmental stimulus, the behavioral output often includes complex, nonlinear response to the input stimulus (Gordus et al., 2015). The incomplete match between the spike-like AFD activity and behavioral components suggest that the signal from a different neuron modulates the encoded environmental stimulus and produces the modified signal for behavioral regulation. The AWC thermosensory neurons are candidates for this role (Biron et al., 2008; Kuhara et al., 2008), and reconstruction and modeling approaches for AWC in combination with our AFD model may provide clues better to understand behavioral output. Another possibility for the incompatibility between sensory activity and behavior is that internal states inside the neural circuit govern the behavioral regulation, and an environmental stimulus is a modulator of the internal state. The pair of AIY interneurons postsynaptic to AFD could integrate other cues that modify the behavioral output, because AIY interneurons are thought to receive signals from both AFD and AWC neurons and have an important role in stimulus-related behavioral regulation, including thermotaxis (Mori and Ohshima, 1995; Kocabas et al., 2012; Li et al., 2014). Simultaneous monitoring and reconstructing approaches for these neurons may clarify the regulatory signal for behavioral regulation. Also, we should consider the properties of signal transduction in the neural circuit, such as the graded signal transmission from AFD to AIY (Narayan et al., 2011). Together with the report on the graded signal transduction in the other sensory-interneuronal connection between ASH and AVA (Lindsay et al., 2011), we speculate that interneurons receive scaled sensory signals to integrate different modes of sensory stimuli. A simulationassisted mathematical modeling approach for several neurons in combination with our model may be helpful to understand the generation of behavioral regulatory signals in response to environmental stimuli.

\section{A modeling approach to study environmental search}

To understand the functional properties of AFD thermosensory neurons during thermotaxis, we took a phenomenological approach, which provides logical identity of the AFD response. The identification of a particular neural response allows us to compare with other identified neural responses at a functional level, by which we can deduce common mechanisms in different sensory systems. An example of comparable neurons is the system identification for C. elegans olfactory neuron AWC and nociceptive neuron ASH of biphasic responses (Kato et al., 2014). Common characters of these sensory neurons, including AFD, are robust phasic response and reliable linearity. Our present study separates the mechanism of the response character and operating range of AFD, the latter of which depends on a memorized cultivated temperature. This separation of functionality implies a unique mechanism of neural response and memory in AFD neuron.

As we have shown in this study, the modeling and reconstruction approach provides a basis to examine questions related to the link between environmental stimuli and behavioral output. Identifying the response properties of each neuron enables patterns of neural responses to be linked to behavioral regulation. Extending this approach may enable us to reconstruct a macroscopic behavior consisting of several behavioral regulatory components: taxis behavior with turnings and runs evoked by specific neural activities. These approaches can also be applied for solving more mechanistic questions, such as how the linear response property of a thermosensory neuron contributes to the efficiency of thermotaxis behavior.

Although linear approximations provide a simple and powerful methodology for identifying the response properties of sensory systems, it is not guaranteed to work for describing the relationship between an external stimulus and physiology. There are several hurdles for assay systems to analyze with linear approximations, such as measurement noise, timescale difference between the external stimulus and encoded signals, and complicated relationships between the external stimulus and encoded signals. We found that the thermosensory neuron AFD well represents the external landscape by approximating with a linear kernel. This application provides an additional experimental platform to dissect sensory encoding mechanisms for migrating organisms.

A modeling approach with the simple nervous system of $C$. elegans may connect neural mechanisms to the behavioral studies at different scales, such as a single cellular system of bacterial chemotaxis (Segall et al., 1986; Masson et al., 2012) or moving wild animals in a natural environment (Edwards et al., 2007; Sims et al., 2008). Because the particular characteristics of navigational control against environmental stimulus are conserved from bacteria to mammals, phenomenological examination should connect the targeted behavioral component among the different species. Elucidation of functional components of neural mechanisms, such as sensory response and transmission between neurons, sheds light on the principle of behavioral regulation by neural circuits in changeable natural environments.

\section{References}

Biron D, Wasserman S, Thomas JH, Samuel AD, Sengupta P (2008) An olfactory neuron responds stochastically to temperature and modulates Caenorhabditis elegans thermotactic behavior. Proc Natl Acad Sci U S A 105:11002-11007. CrossRef Medline

Brenner S (1974) The genetics of Caenorhabditis elegans. Genetics 77:71-94. Medline

Celani A, Vergassola M (2010) Bacterial strategies for chemotaxis response. Proc Natl Acad Sci U S A 107:1391-1396. CrossRef Medline

Clark DA, Biron D, Sengupta P, Samuel AD (2006) The AFD sensory neurons encode multiple functions underlying thermotactic behavior in Caenorhabditis elegans. J Neurosci 26:7444-7451. CrossRef Medline

Edelstein A, Amodaj N, Hoover K, Vale R, Stuurman N (2010) Computer control of microscopes using $\mu$ Manager. Curr Protoc Mol Biol Chapter 14:Unit14.20. CrossRef Medline

Edwards AM, Phillips RA, Watkins NW, Freeman MP, Murphy EJ, Afanasyev 
V, Buldyrev SV, da Luz MG, Raposo EP, Stanley HE, Viswanathan GM (2007) Revisiting Lévy flight search patterns of wandering albatrosses, bumblebees and deer. Nature 449:1044-1048. CrossRef Medline

Gomez-Marin A, Stephens GJ, Louis M (2011) Active sampling and decision making in Drosophila chemotaxis. Nat Commun 2:441. CrossRef Medline

Gordus A, Pokala N, Levy S, Flavell SW, Bargmann CI (2015) Feedback from network states generates variability in a probabilistic olfactory circuit. Cell 161:215-227. CrossRef Medline

Hedgecock EM, Russell RL (1975) Normal and mutant thermotaxis in the nematode Caenorhabditis elegans. Proc Natl Acad Sci U S A 72: 4061-4065. CrossRef Medline

Hogg RC, Buisson B, Bertrand D (2005) Allosteric modulation of ligandgated ion channels. Biochem Pharmacol 70:1267-1276. CrossRef Medline

Iino Y, Yoshida K (2009) Parallel use of two behavioral mechanisms for chemotaxis in Caenorhabditis elegans. Neuroscience 29:5370-5380. CrossRef Medline

Kato S, Xu Y, Cho CE, Abbott LF, Bargmann CI (2014) Temporal responses of C. elegans chemosensory neurons are preserved in behavioral dynamics. Neuron 81:616-628. CrossRef Medline

Kim E, Sun L, Gabel CV, Fang-Yen C (2013) Long-term imaging of Caenorhabditis elegans using nanoparticle-mediated immobilization. PLoS One 8:e53419. CrossRef Medline

Kimura KD, Miyawaki A, Matsumoto K, Mori I (2004) The C. elegans thermosensory neuron AFD responds to warming. Curr Biol 14:1291-1295. CrossRef Medline

Kocabas A, Shen CH, Guo ZV, Ramanathan S (2012) Controlling interneuron activity in Caenorhabditis elegans to evoke chemotactic behaviour. Nature 490:273-277. CrossRef Medline

Kodama E, Kuhara A, Mohri-Shiomi A, Kimura KD, Okumura M, Tomioka M, Iino Y, Mori I (2006) Insulin-like signaling and the neural circuit for integrative behavior in C. elegans. Genes Dev 20:2955-2960. CrossRef Medline

Kuhara A, Okumura M, Kimata T, Tanizawa Y, Takano R, Kimura KD, Inada H, Matsumoto K, Mori I (2008) Temperature sensing by an olfactory neuron in a circuit controlling behavior of C. elegans. Science 320: 803-807. CrossRef Medline

Kuhara A, Ohnishi N, Shimowada T, Mori I (2011) Neural coding in a single sensory neuron controlling opposite seeking behaviours in Caenorhabditis elegans. Nat Commun 2:355. CrossRef Medline

Lagarias JC, Reeds JA, Wright MH, Wright PE (1998) Convergence properties of the Nelder-Mead simplex method in low dimensions. SIAM J Optim 9:112-147. CrossRef

Li Z, Liu J, Zheng M, Xu XZ (2014) Encoding of both analog- and digitallike behavioral outputs by one C. elegans interneuron. Cell 159:751-765. CrossRef Medline

Lindsay TH, Thiele TR, Lockery SR (2011) Optogenetic analysis of synaptic transmission in the central nervous system of the nematode Caenorhabditis elegans. Nat Commun 2:306. CrossRef Medline
Luo L, Cook N, Venkatachalam V, Martinez-Velazquez LA, Zhang X, Calvo AC, Hawk J, MacInnis BL, Frank M, Ng JH, Klein M, Gershow M, Hammarlund M, Goodman MB, Colón-Ramos DA, Zhang Y, Samuel AD (2014) Bidirectional thermotaxis in Caenorhabditis elegans is mediated by distinct sensorimotor strategies driven by the AFD thermosensory neurons. Proc Natl Acad Sci U S A 111:2776-2781. CrossRef Medline

Masson JB, Voisinne G, Wong-Ng J, Celani A, Vergassola M (2012) Noninvasive inference of the molecular chemotactic response using bacterial trajectories. Proc Natl Acad Sci U S A 109:1802-1807. CrossRef Medline

Mello CC, Kramer JM, Stinchcomb D, Ambros V (1991) Efficient gene transfer in C. elegans: extrachromosomal maintenance and integration of transforming sequences. EMBO J 10:3959-3970. Medline

Mohri A, Kodama E, Kimura KD, Koike M, Mizuno T, Mori I (2005) Genetic control of temperature preference in the nematode Caenorhabditis elegans. Genetics 169:1437-1450. Medline

Mori I, Ohshima Y (1995) Neural regulation of thermotaxis in Caenorhabditis elegans. Nature 376:344-348. CrossRef Medline

Narayan A, Laurent G, Sternberg PW (2011) Transfer characteristics of a thermosensory synapse in Caenorhabditis elegans. Proc Natl Acad Sci U S A 108:9667-9672. CrossRef Medline

Ramot D, MacInnis BL, Goodman MB (2008) Bidirectional temperaturesensing by a single thermosensory neuron in C. elegans. Nat Neurosci 11:908-915. CrossRef Medline

Rieke F, Warland D, van Steveninch R de R, Bialek W (1999) Spikes-exploring the neural code. Cambridge, MA: Massachusetts Institute of Technology.

Ryu WS, Samuel AD (2002) Thermotaxis in Caenorhabditis elegans analyzed by measuring responses to defined thermal stimuli. J Neurosci 22: 5727-5733. Medline

Satterlee JS, Sasakura H, Kuhara A, Berkeley M, Mori I, Sengupta P (2001) Specification of thermosensory neuron fate in C. elegans requires ttx-1, a homolog of otd/Otx. Neuron 31:943-956. CrossRef Medline

Segall JE, Block SM, Berg HC (1986) Temporal comparisons in bacterial chemotaxis. Proc Natl Acad Sci U S A 83:8987-8991. CrossRef Medline

Sims DW, Southall EJ, Humphries NE, Hays GC, Bradshaw CJ a, Pitchford JW, James A, Ahmed MZ, Brierley AS, Hindell MA, Morritt D, Musyl MK, Righton D, Shepard EL, Wearmouth VJ, Wilson RP, Witt MJ, Metcalfe JD (2008) Scaling laws of marine predator search behaviour. Nature 451: 1098-1102. CrossRef Medline

Suzuki H, Thiele TR, Faumont S, Ezcurra M, Lockery SR, Schafer WR (2008) Functional asymmetry in Caenorhabditis elegans taste neurons and its computational role in chemotaxis. Nature 454:114-117. CrossRef Medline

Wang Z, Bovik AC, Sheikh HR, Simoncelli EP (2004) Image quality assessment: from error visibility to structural similarity. IEEE Trans Image Process 13:600-612. CrossRef Medline

Zariwala HA, Miller AC, Faumont S, Lockery SR (2003) Step response analysis of thermotaxis in Caenorhabditis elegans. J Neurosci 23:4369-4377. Medline 